\title{
Spectroscopy of singly, doubly, and triply bottom baryons
}

\author{
Ke-Wei Wei ${ }^{1,4, *}$, Bing Chen ${ }^{1, \dagger}$, Na Liu ${ }^{1,3}$, Qian-Qian Wang ${ }^{1}$, and Xin-Heng Guo ${ }^{2, \ddagger}$ \\ ${ }^{1}$ College of Physics and Electrical Engineering, Anyang Normal University, Anyang 455000, China \\ ${ }^{2}$ College of Nuclear Science and Technology, Beijing Normal University, Beijing 100875, China \\ ${ }^{3}$ School of Physics and Technology, Nanjing Normal University, Nanjing 210023, China \\ 4 Theoretical Physics Center for Science Facilities, CAS, Beijing 100049, China
}

(Dated: September 28, 2018)

\begin{abstract}
Recently, some singly bottom baryons have been established experimentally, but none of doubly or triply bottom baryons has been observed. Under the Regge phenomenology, the mass of a ground state unobserved doubly or triply bottom baryon is expressed as a function of masses of the well established light baryons and singly bottom baryons. Then, the values of Regge slopes and Regge intercepts for baryons containing one, two, or three bottom quarks are calculated. After that, masses of the orbitally excited singly, doubly, and triply bottom baryons are estimated. Our predictions may be useful for the discovery of these baryons and the $J^{P}$ assignment of them.
\end{abstract}

PACS numbers: 11.55.Jy, 14.20.Mr, 12.10.Kt, 12.40.Yx, 12.40.Nn

\footnotetext{
* e-mail: weikw@hotmail.com

$\dagger$ e-mail: chenbing@shu.edu.cn

$\ddagger$ Corresponding author, e-mail: xhguo@bnu.edu.cn
} 


\section{INTRODUCTION}

According to the Particle Data Group's latest "Review of Particle Physics" (RPP) [1], some singly bottom baryons $\left(\Lambda_{b}, \Lambda_{b}(5912), \Lambda_{b}(5920), \Sigma_{b}, \Sigma_{b}^{*}, \Xi_{b}, \Xi_{b}^{\prime}, \Xi_{b}^{*}\right.$, and $\left.\Omega_{b}\right)$ have been established. However, none of doubly or triply bottom baryons has been observed until now. Therefore, in the present work, we will focus on searching mass relations which can be used to express the mass of a doubly or triply bottom baryon as a function of masses of the well established baryons. This is the main motivation of this work.

It is noted that the Gell-Mann-Okubo formula [2] cannot be directly applied to the charmed and bottom hadrons due to higher-order breaking effects. The Regge trajectory ansatz is an effective phenomenological model to study mass relations [3- 7] and mass spectra [8 15] for mesons and baryons. In a previous work [4], a way was proposed to express the mass of a doubly charmed baryons as a function of masses of the well established light baryons and singly charmed baryons. In the present work, under Regge phenomenology, we will express the mass of an unobserved ground state (the orbital quantum number $L=0$ ) baryon containing one, two or three bottom quarks as a function of masses of the well established light baryons and singly bottom baryons. The mass values will be given and compared with those obtained in many other approaches [7, 8, 16 62 , 64].

The slopes and intercepts of Regge trajectories are useful for many spectral and nonspectral purposes [3, 11, 65], for example, in the fragmentation [66] and recombination [67] models. Therefore, Regge slopes and Regge intercepts are fundamental constants of hadronic dynamics, perhaps more important than the masses of particular states [68]. Thus, the determination of Regge slopes and intercepts of hadrons is of great importance since this affords opportunities for a better understanding of the dynamics of strong interactions in the processes of production of charmed and bottom hadrons at high energies and estimates of their production rates [65]. In our previous work in 2008 [3], the numerical values of Regge slopes and Regge intercepts for the $\frac{1}{2}^{+}$and $\frac{3}{2}^{+}$light and charmed baryon trajectories are extracted. In Ref. [11], Li et al. gave the values of Regge slopes and intercepts for all $S U(5)$ mesons (involving the $u, d, s, c, b$ quarks). In Ref. [8], Ebert et al. gave the values of Regge slopes for singly charmed and singly bottom baryons. In the present work, we will extract Regge slopes and Regge intercepts for the singly, doubly, and triply bottom baryons and calculate masses of the orbitally excited baryons $(L=1,2,3,4)$ lying on these Regge trajectories.

The remainder of this paper is organized as follows. In Sec. II, under Regge phenomenology, masses of the unobserved ground state singly, doubly, and triply bottom baryons $\Omega_{b}^{*}$, 
$\Omega_{b b}^{*}, \Omega_{b b}, \Xi_{b b}^{*}, \Xi_{b b}$, and $\Omega_{b b b}$ will be given. In Sec. III, we will calculate Regge slopes and Regge intercepts for the $\frac{1}{2}^{+}$and $\frac{3}{2}^{+}$singly, doubly, and triply bottom baryon trajectories. After that, masses of the orbitally excited baryons lying on these trajectories will be estimated. In Sec. IV, a short discussion and conclusion will be given.

\section{MASSES OF THE UNOBSERVED $\Omega_{b}^{*}, \Omega_{b b}^{(*)}, \Xi_{b b}^{(*)}$, AND $\Omega_{b b b}$ BARYONS}

In this section, we will first give a short introduction for Regge phenomenology and express the quadratic masses of the ground state unobserved $\Omega_{b}^{*}, \Omega_{b b}^{(*)}, \Xi_{b b}^{(*)}$, and $\Omega_{b b b}$ baryons as functions of the quadratic masses of the well established light baryons and singly bottom baryons. After that, the mass values will be given and compared.

\section{A. Regge phenomenology}

Regge theory is concerned with almost all aspects of strong interactions, including the particle spectra, the high energy behavior of scattering amplitudes, and the forces between particles [69]. It is known from Regge theory that all mesons and baryons are associated with Regge trajectories (Regge poles which move in the complex angular momentum plane as a function of energy) [70]. Hadrons lying on the same Regge trajectory which have the same internal quantum numbers are classified into the same family [69, 71]. Regge trajectories for hadrons can be parameterized as follows:

$$
J=\alpha(M)=a(0)+\alpha^{\prime} M^{2}
$$

where $\alpha^{\prime}$ and $a(0)$ are respectively the slope and intercept of the Regge trajectory on which the particles lie. For a baryon multiplet, Regge intercepts and Regge slopes for different flavors can be related by the following relations (see Ref. [3] and references therein):

$$
\begin{aligned}
a_{i i k}(0)+a_{j j k}(0) & =2 a_{i j k}(0), \\
\frac{1}{\alpha_{i i k}^{\prime}}+\frac{1}{\alpha_{j j k}^{\prime}} & =\frac{2}{\alpha_{i j k}^{\prime}}
\end{aligned}
$$


where $i, j$, and $k$ denote arbitrary light or heavy quarks. Based on Eqs. (2) and (3) , one can introduce two parameters $\gamma_{x}$ and $\lambda_{x}$,

$$
\begin{gathered}
\gamma_{x}=\frac{1}{\alpha_{n n x}^{\prime}}-\frac{1}{\alpha_{n n n}^{\prime}}, \\
\lambda_{x}=a_{n n n}(0)-a_{n n x}(0),
\end{gathered}
$$

where $n$ represents the light unflavored quark $u$ or $d, x$ denotes $i, j$, or $k$. Therefore,

$$
\begin{aligned}
a_{i j k}(0) & =a_{n n n}(0)-\lambda_{i}-\lambda_{j}-\lambda_{k}, \\
\frac{1}{\alpha_{i j k}^{\prime}} & =\frac{1}{\alpha_{n n n}^{\prime}}+\gamma_{i}+\gamma_{j}+\gamma_{k} .
\end{aligned}
$$

From Eq. (11), one can have

$$
\begin{gathered}
J=a_{n n n}(0)+\alpha_{n n n}^{\prime} M_{n n n}^{2} \\
J=a_{i j k}(0)+\alpha_{i j k}^{\prime} M_{i j k}^{2} .
\end{gathered}
$$

From Eqs. (6) - (9) , we can have

$$
M_{i j k}^{2}=\left(\alpha_{n n n}^{\prime} M_{n n n}^{2}+\lambda_{i}+\lambda_{j}+\lambda_{k}\right)\left(\frac{1}{\alpha_{n n n}^{\prime}}+\gamma_{i}+\gamma_{j}+\gamma_{k}\right)
$$

As demonstrated in Ref. [3], in order to evaluate the high-order effects, we introduce the parameter $\delta$,

$$
\delta_{i j, q} \equiv M_{i i q}^{2}+M_{j j q}^{2}-2 M_{i j q}^{2}
$$

where $q$ is an arbitrary light or heavy quark. Combining Eqs. (10) and (11), we can prove

$$
\delta_{i j, q}=M_{i i q}^{2}+M_{j j q}^{2}-2 M_{i j q}^{2}=2\left(\lambda_{i}-\lambda_{j}\right)\left(\gamma_{i}-\gamma_{j}\right)
$$

From Eq. (12), one can see that $\delta_{i j, q}$ is independent of the $q$ quark.

\section{B. Mass expressions and masses for the $\frac{3}{2}^{+} \Omega_{b}^{*}, \Omega_{b b}^{*}, \Xi_{b b}^{*}$, and $\Omega_{b b b}$ baryons}

For the $\frac{3}{2}^{+}$multiplet, noticing that $\delta_{i j, q}^{\frac{3}{2}^{+}}$is independent of $q$ in the above relation (12), when $i=n, j=s, q=s, c, b$, Eqs. (12) can be expressed as

$$
\delta_{n s, q}^{\frac{3}{2}}=M_{\Sigma^{*}}^{2}+M_{\Omega}^{2}-2 M_{\Xi^{*}}^{2}=M_{\Sigma_{c}^{*}}^{2}+M_{\Omega_{c}^{*}}^{2}-2 M_{\Xi_{c}^{*}}^{2}=M_{\Sigma_{b}^{*}}^{2}+M_{\Omega_{b}^{*}}^{2}-2 M_{\Xi_{b}^{*}}^{2}
$$


When $i=s, j=b, q=n, s, b$, Eqs. (12) can be expressed as

$$
\delta_{s b, q}^{\frac{3}{2}+}=M_{\Xi^{*}}^{2}+M_{\Xi_{b b}^{*}}^{2}-2 M_{\Xi_{b}^{*}}^{2}=M_{\Omega}^{2}+M_{\Omega_{b b}^{*}}^{2}-2 M_{\Omega_{b}^{*}}^{2}=M_{\Omega_{b}^{*}}^{2}+M_{\Omega_{b b b}}^{2}-2 M_{\Omega_{b b}^{*}}^{2}
$$

Using Eqs. (11) and (2), one can obtain

$$
\alpha_{i i k}^{\prime} M_{i i k}^{2}+\alpha_{j j k}^{\prime} M_{j j k}^{2}=2 \alpha_{i j k}^{\prime} M_{i j k}^{2}
$$

With Eqs. (3) and (15), when the quark masses satisfy $m_{j}>m_{i}$, we can obtain [3]

$$
\frac{\alpha_{j j k}^{\prime}}{\alpha_{i i k}^{\prime}}=\frac{1}{2 M_{j j k}^{2}} \times\left[\left(4 M_{i j k}^{2}-M_{i i k}^{2}-M_{j j k}^{2}\right)+\sqrt{\left(4 M_{i j k}^{2}-M_{i i k}^{2}-M_{j j k}^{2}\right)^{2}-4 M_{i i k}^{2} M_{j j k}^{2}}\right] .
$$

Therefore, for the $\frac{3}{2}^{+}$multiplet,

$$
\begin{aligned}
\frac{\alpha_{b b s}^{\prime}}{\alpha_{n n s}^{\prime}} & =\frac{1}{2 M_{\Omega_{b b}^{*-}}^{2}} \times\left[\left(4 M_{\Xi_{b}^{* 0}}^{2}-M_{\Sigma^{*+}}^{2}-M_{\Omega_{b b}^{*-}}^{2}\right)+\sqrt{\left(4 M_{\Xi_{b}^{* 0}}^{2}-M_{\Sigma^{*+}}^{2}-M_{\Omega_{b b}^{*-}}^{2}\right)^{2}-4 M_{\Sigma^{*+}}^{2} M_{\Omega_{b b}^{*-}}^{2}}\right], \\
\frac{\alpha_{b b s}^{\prime}}{\alpha_{s s s}^{\prime}} & =\frac{1}{2 M_{\Omega_{b b}^{*-}}^{2}} \times\left[\left(4 M_{\Omega_{b}^{*-}}^{2}-M_{\Omega^{-}}^{2}-M_{\Omega_{b b}^{*-}}^{2}\right)+\sqrt{\left(4 M_{\Omega_{b}^{*-}}^{2}-M_{\Omega^{-}}^{2}-M_{\Omega_{b b}^{*-}}^{2}\right)^{2}-4 M_{\Omega^{-}}^{2} M_{\Omega_{b b}^{*-}}^{2}}\right], \\
\frac{\alpha_{s s s}^{\prime}}{\alpha_{n n s}^{\prime}} & =\frac{1}{2 M_{\Omega^{-}}^{2}} \times\left[\left(4 M_{\Xi^{* 0}}^{2}-M_{\Sigma^{*+}}^{2}-M_{\Omega^{-}}^{2}\right)+\sqrt{\left(4 M_{\Xi^{* 0}}^{2}-M_{\Sigma^{*+}}^{2}-M_{\Omega^{-}}^{2}\right)^{2}-4 M_{\Sigma^{*+}}^{2} M_{\Omega^{-}}^{2}}\right] .
\end{aligned}
$$

With the identical equation $\frac{\alpha_{b b s}^{\prime}}{\alpha_{n n s}^{\prime}} \equiv \frac{\alpha_{b b s}^{\prime}}{\alpha_{s s s}^{\prime}} \times \frac{\alpha_{s s s}^{\prime}}{\alpha_{n n s}^{\prime}}$, one can have

$$
\begin{aligned}
& \frac{1}{2 M_{\Omega_{b b}^{*}}^{2}} \times\left[\left(4 M_{\Xi_{b}^{*}}^{2}-M_{\Sigma^{*}}^{2}-M_{\Omega_{b b}^{*}}^{2}\right)+\sqrt{\left(4 M_{\Xi_{b}^{*}}^{2}-M_{\Sigma^{*}}^{2}-M_{\Omega_{b b}^{*}}^{2}\right)^{2}-4 M_{\Sigma^{*}}^{2} M_{\Omega_{b b}^{*}}^{2}}\right] \\
= & \frac{1}{2 M_{\Omega_{b b}^{*}}^{2}} \times\left[\left(4 M_{\Omega_{b}^{*}}^{2}-M_{\Omega}^{2}-M_{\Omega_{b b}^{*}}^{2}\right)+\sqrt{\left(4 M_{\Omega_{b}^{*}}^{2}-M_{\Omega}^{2}-M_{\Omega_{b b}^{*}}^{2}\right)^{2}-4 M_{\Omega^{2}}^{2} M_{\Omega_{b b}^{*}}^{2}}\right] \\
& \times \frac{1}{2 M_{\Omega}^{2}} \times\left[\left(4 M_{\Xi^{*}}^{2}-M_{\Sigma^{*}}^{2}-M_{\Omega}^{2}\right)+\sqrt{\left(4 M_{\Xi^{*}}^{2}-M_{\Sigma^{*}}^{2}-M_{\Omega}^{2}\right)^{2}-4 M_{\Sigma^{*}}^{2} M_{\Omega^{2}}^{2}}\right] .
\end{aligned}
$$

In the following, we will give the mass expressions and mass values for the ground state unobserved bottom baryons. Before this, we will apply the method first to the known $\Omega_{c}^{*}$ in order to see how well it works. From Eq. (13), we can obtain the squared masse expression for $\Omega_{c}^{*}$ as a function of the squared masses of $\Sigma^{*}, \Xi^{*}, \Omega, \Sigma_{c}^{*}$, and $\Xi_{c}^{*}$,

$$
M_{\Omega_{c}^{*}}^{2}=M_{\Sigma^{*}}^{2}+M_{\Omega}^{2}-2 M_{\Xi^{*}}^{2}-M_{\Sigma_{c}^{*}}^{2}+2 M_{\Xi_{c}^{*}}^{2}
$$


From the latest RPP [1], $M_{\Sigma^{*}}=1385.0 \pm 2.3 \mathrm{MeV}, M_{\Xi^{*}}=1533.4 \pm 1.7 \mathrm{MeV}, M_{\Omega}=$ $1672.45 \pm 0.29 \mathrm{MeV}, M_{\Sigma_{c}^{*}}=2518.1 \pm 0.9 \mathrm{MeV}, M_{\Xi_{c}^{*}}=2645.9 \pm 0.5 \mathrm{MeV}$. Inserting these mass values into the relation (19), one can have $M_{\Omega_{c}^{*}}=2769.8 \pm 2.6 \mathrm{MeV}$. In the latest $\operatorname{RPP}$ [1], $M_{\Omega_{c}^{*}}=2765.9 \pm 2.0 \mathrm{MeV}$. Our result for $M_{\Omega_{c}^{*}}$ is in reasonable agreement with the experimental value. In the following we will apply the method to bottom baryons.

From Eq. (13), we can obtain the squared mass of $\Omega_{b}^{*}$ as a function of the squared masses of $\Sigma^{*}, \Xi^{*}, \Omega, \Sigma_{b}^{*}$, and $\Xi_{b}^{*}$,

$$
M_{\Omega_{b}^{*}}^{2}=M_{\Sigma^{*}}^{2}+M_{\Omega}^{2}-2 M_{\Xi^{*}}^{2}-M_{\Sigma_{b}^{*}}^{2}+2 M_{\Xi_{b}^{*}}^{2}
$$

From Eqs. (18) and (20), we can obtain the squared mass of $\Omega_{b b}^{*}$ as a function of the squared masses of $\Sigma^{*}, \Xi^{*}, \Omega, \Sigma_{b}^{*}$, and $\Xi_{b}^{*}$,

$$
\begin{aligned}
M_{\Omega_{b b}^{*}}^{2}= & 5 M_{\Xi_{b}^{*}}^{2}-2 M_{\Xi^{*}}^{2}-M_{\Sigma_{b}^{*}}^{2}+\frac{M_{\Omega}^{2}}{2}+\frac{5 M_{\Sigma^{*}}^{2}}{2}-\frac{2\left(M_{\Xi_{b}^{*}}^{2}-M_{\Sigma_{b}^{*}}^{2}\right)\left(M_{\Xi^{*}}^{2}-M_{\Sigma^{*}}^{2}\right)}{M_{\Omega}^{2}-2 M_{\Xi^{*}}^{2}+M_{\Sigma^{*}}^{2}}+\frac{\sqrt{M_{\Omega}^{4}-2 M_{\Omega}^{2}\left(4 M_{\Xi^{*}}^{2}+M_{\Sigma^{*}}^{2}\right)+\left(M_{\Sigma^{*}}^{2}-4 M_{\Xi^{*}}^{2}\right)^{2}}}{2\left(M_{\Omega}^{2}-2 M_{\Xi^{*}}^{2}+M_{\Sigma^{*}}^{2}\right)} \\
& \times \sqrt{\left(M_{\Omega}^{2}-2 M_{\Xi^{*}}^{2}+M_{\Sigma^{*}}^{2}\right)\left(M_{\Omega}^{2}-4 M_{\Xi_{b}^{*}}^{2}-2 M_{\Xi^{*}}^{2}-4 M_{\Sigma_{b}^{*}}^{2}+M_{\Sigma^{*}}^{2}\right)+4\left(M_{\Xi_{b}^{*}}^{2}-M_{\Sigma_{b}^{*}}^{2}\right)^{2}} .
\end{aligned}
$$

From Eqs. (14), (20), and (21), we can obtain the mass expressions for $\Xi_{b b}^{*}$ and $\Omega_{b b b}$.

$$
\begin{aligned}
& M_{\Xi_{b b}^{*}}^{2}= 3 M_{\Xi_{b}^{*}}^{2}+M_{\Xi^{*}}^{2}+M_{\Sigma_{b}^{*}}^{2}+\frac{M_{\Sigma^{*}}^{2}}{2}-\frac{M_{\Omega}^{2}}{2}-\frac{2\left(M_{\Xi_{b}^{*}}^{2}-M_{\Sigma_{b}^{*}}^{2}\right)\left(M_{\Xi^{*}}^{2}-M_{\Sigma^{*}}^{2}\right)}{M_{\Omega}^{2}-2 M_{\Xi^{*}}^{2}+M_{\Sigma^{*}}^{2}}+\frac{\sqrt{M_{\Omega}^{4}-2 M_{\Omega}^{2}\left(4 M_{\Xi^{*}}^{2}+M_{\Sigma^{*}}^{2}\right)+\left(M_{\Sigma^{*}}^{2}-4 M_{\Xi^{*}}^{2}\right)^{2}}}{2\left(M_{\Omega}^{2}-2 M_{\Xi^{*}}^{2}+M_{\Sigma^{*}}^{2}\right)} \\
& \times \sqrt{\left(M_{\Omega}^{2}-2 M_{\Xi^{*}}^{2}+M_{\Sigma^{*}}^{2}\right)\left(M_{\Omega}^{2}-4 M_{\Xi_{b}^{*}}^{2}-2 M_{\Xi^{*}}^{2}-4 M_{\Sigma_{b}^{*}}^{2}+M_{\Sigma^{*}}^{2}\right)+4\left(M_{\Xi_{b}^{*}}^{2}-M_{\Sigma_{b}^{*}}^{2}\right)^{2}} \\
& M_{\Omega_{b b b}}^{2}= 9 M_{\Xi_{b}^{*}}^{2}+\frac{9 M_{\Sigma^{*}}^{2}}{2}-\frac{M_{\Omega}^{2}}{2}-\frac{6\left(M_{\Xi_{b}^{*}}^{2}-M_{\Sigma_{b}^{*}}^{2}\right)\left(M_{\Xi^{*}}^{2}-M_{\Sigma^{*}}^{2}\right)}{M_{\Omega}^{2}-2 M_{\Xi^{*}}^{2}+M_{\Sigma^{*}}^{2}}+\frac{\sqrt{-2 M_{\Omega}^{2}\left(4 M_{\Xi^{*}}^{2}+M_{\Sigma^{*}}^{2}\right)+M_{\Omega}^{4}+\left(M_{\Sigma^{*}}^{2}-4 M_{\Xi^{*}}^{2}\right)^{2}}}{2\left(M_{\Omega}^{2}-2 M_{\Xi^{*}}^{2}+M_{\Sigma^{*}}^{2}\right) / 3} \\
& \times \sqrt{\left(M_{\Omega}^{2}-2 M_{\Xi^{*}}^{2}+M_{\Sigma^{*}}^{2}\right)\left(M_{\Omega}^{2}-4 M_{\Xi_{b}^{*}}^{2}-2 M_{\Xi^{*}}^{2}-4 M_{\Sigma_{b}^{*}}^{2}+M_{\Sigma^{*}}^{2}\right)+4\left(M_{\Xi_{b}^{*}}^{2}-M_{\Sigma_{b}^{*}}^{2}\right)^{2}}
\end{aligned}
$$

From the latest RPP [1], $M_{\Sigma^{*}}=1385.0 \pm 2.3 \mathrm{MeV}, M_{\Xi^{*}}=1533.4 \pm 1.7 \mathrm{MeV}, M_{\Omega}=$ $1672.45 \pm 0.29 \mathrm{MeV}, M_{\Sigma_{b}^{*}}=5833.6 \pm 2.4 \mathrm{MeV}, M_{\Xi_{b}^{*}}=5952.1 \pm 3.3 \mathrm{MeV}$. Inserting the corresponding mass values into the relation (20), one can have $M_{\Omega_{b}^{*}}=6069.4 \pm 6.9 \mathrm{MeV}$. Therefore, $M_{\Omega_{b}^{*}}-M_{\Omega_{b}}=21.4 \pm 7.2 \mathrm{MeV}$, which is close to the experimental data, $M_{\Sigma_{b}^{*}}-M_{\Sigma_{b}}$ $=21.2 \pm 2.0 \mathrm{MeV}$ and $M_{\Xi_{b}^{*-}}-M_{\Xi_{b}^{\prime-}}=20.3 \pm 0.1 \mathrm{MeV}$, as it should be. The prediction, $M_{\Omega_{b}^{*}}-M_{\Omega_{b}}=21.4 \pm 7.2 \mathrm{MeV}$, is in reasonable agreement with the value $(24.0 \pm 0.7 \mathrm{MeV})$ given in Ref. [62]. Inserting the above mass values of $\Sigma^{*}, \Xi^{*}, \Omega, \Sigma_{b}^{*}$, and $\Xi_{b}^{*}$ into the relation (21), one can get $M_{\Omega_{b b}^{*}}=10431 \pm 40 \mathrm{MeV}$. From Eq. (23) one has $M_{\Omega_{b b b}}=14788 \pm$ $80 \mathrm{MeV}$. Similarly, we can obtain the expression for $M_{\Xi_{b b}^{*}}$ and get its value to be $10316.3 \pm$ $37.8 \mathrm{MeV}(10316 \pm 38 \mathrm{MeV}$ when truncated to the $1 \mathrm{MeV}$ digit). Comparison of the masses 
of $\Omega_{b}^{*}, \Omega_{b b}^{*}, \Omega_{b b b}$, and $\Xi_{b b}^{*}$ extracted in the present work and those given in other references is shown in Table 1.

\section{Masses of the ground unobserved $\frac{1}{2}^{+} \Omega_{b b}$ and $\Xi_{b b}$ baryons}

Based on Eqs. (13) and (14), one can have

$$
\left(M_{\Omega_{b b}^{*}}^{2}-M_{\Xi_{b b}^{*}}^{2}\right)+\left(M_{\Xi^{*}}^{2}-M_{\Sigma^{*}}^{2}\right)=\left(M_{\Omega_{b}^{*}}^{2}-M_{\Sigma_{b}^{*}}^{2}\right) .
$$

For the $\frac{1}{2}^{+}$multiplet, one can have a mass relation similar to Eq. (24),

$$
\left(M_{\Omega_{b b}}^{2}-M_{\Xi_{b b}}^{2}\right)+\left(M_{\Xi}^{2}-M_{\Sigma}^{2}\right)=\left(M_{\Omega_{b}}^{2}-M_{\Sigma_{b}}^{2}\right) .
$$

The linear forms in Eqs. (24) and (25) can satisfy the instanton model [72] and the SU(8) symmetry [73].

For the $\frac{1}{2}^{+}$multiplet, based on Eq. (12),$\delta_{n b, s}^{\frac{1}{2}^{+}}+\delta_{s b, n}^{\frac{1}{2}^{+}}$can be expressed as

$$
\delta_{n b, s}^{\frac{1}{2}^{+}}+\delta_{s b, n}^{\frac{1}{2}^{+}}=M_{\Sigma}^{2}+M_{\Omega_{b b}}^{2}-2\left(\frac{3 M_{\Xi_{b}}^{2}+M_{\Xi_{b}^{\prime}}^{2}}{4}\right)+M_{\Xi}^{2}+M_{\Xi_{b b}}^{2}-2\left(\frac{3 M_{\Xi_{b}}^{2}+M_{\Xi_{b}^{\prime}}^{2}}{4}\right) .
$$

In Ref. [3], it was pointed out that the values of $\delta_{i j}$ are only a little different between different multiplets for the same $i$ and $j$, although $\delta_{i j}$ are very sensitive to different quark flavors $i$ or $j$. As done in Refs. [3, 44], assuming that $\delta_{i j}^{\frac{1}{2}^{+}}=\delta_{i j}^{\frac{3}{2}^{+}}$, we have $\delta_{n b}^{\frac{1}{2}^{+}}+\delta_{s b}^{\frac{1}{2}^{+}}=\delta_{n b}^{\frac{3}{2}^{+}}+\delta_{s b}^{\frac{3}{2}^{+}}$. The expression for $\delta_{s b}^{\frac{3}{2}}$ has been given in Eq. (14). Based on Eq. (12), when $i=n, j=b, q=s$, $\delta_{n b, s}^{\frac{3^{+}}{2}}$ can be expressed as

$$
\delta_{n b, s}^{\frac{3}{2}+}=M_{\Sigma^{*}}^{2}+M_{\Omega_{b b}^{*}}^{2}-2 M_{\Xi_{b}^{*}}^{2}
$$

With Eqs. (14), (25), (26) , and (27), we can obtain the expressions for $M_{\Omega_{b b}}$ and $M_{\Xi_{b b}}$. Then, inserting the masses of $\Sigma, \Xi, \Sigma_{b}, \Xi_{b}, \Xi_{b}^{\prime}, \Omega_{b}, \Sigma^{*}, \Omega, \Xi_{b}^{*}$ from RPP [1] and $\Omega_{b}^{*}, \Omega_{b b}^{*}$ obtained in Sec. II, we can get the following values: $M_{\Omega_{b b}}=10320 \pm 37 \mathrm{MeV}, M_{\Xi_{b b}}=10199 \pm 37 \mathrm{MeV}$. Comparison of the masses of $\Xi_{b b}$ and $\Omega_{b b}$ extracted in the present work and those given in other references is also shown in Table 1. 
Table 1. The masses of the ground state unobserved singly, doubly, and triply bottom baryons (in units of $\mathrm{MeV}$ ). Our results are labeled with "Pre". The isospin splittings, $M_{\Xi_{b b}^{-}}-M_{\Xi_{b b}^{0}}=2.3 \pm 0.7 \mathrm{MeV}, M_{\Xi_{b b}^{*-}}-M_{\Xi_{b b}^{* 0}}=1.6 \pm 0.6 \mathrm{MeV}$.

\begin{tabular}{|c|c|c|c|c|c|c|}
\hline & $\Omega_{b}^{*}$ & $\Xi_{b b}$ & $\Omega_{b b}$ & $\Xi_{b b}^{*}$ & $\Omega_{b b}^{*}$ & $\Omega_{b b b}$ \\
\hline Pre & $6069.4 \pm 6.9$ & $10199 \pm 37$ & $10320 \pm 37$ & $10316 \pm 38$ & $10431 \pm 40$ & $14788 \pm 80$ \\
\hline [16] & $6170 \pm 150$ & $10170 \pm 140$ & $10320 \pm 140$ & $10220 \pm 150$ & $10380 \pm 140$ & $14830 \pm 100$ \\
\hline [17] & 6051 & 10003 & 10142 & 10048 & 10181 & 14248 \\
\hline [18] & 6102 & 10130 & 10422 & 10144 & 10432 & 14569 \\
\hline [19] & $6000 \pm 160$ & $9780 \pm 70$ & $9850 \pm 70$ & $10350 \pm 80$ & $10280 \pm 50$ & $13280 \pm 100$ \\
\hline [20] & 6102 & 10340 & 10454 & 10367 & 10486 & 14834 \\
\hline [21] & & 10093 & 10133 & 10180 & 10200 & \\
\hline [22] & 6088 & 10202 & 10359 & 10237 & 10389 & \\
\hline [23] & $6035 \pm 60$ & & & $10250 \pm 120$ & $10395 \pm 120$ & $14760 \pm 180$ \\
\hline [24] & $6090 \pm 50$ & $10340 \pm 100$ & $10370 \pm 100$ & $10370 \pm 100$ & $10400 \pm 100$ & \\
\hline [25] & & 10272 & 10369 & 10337 & 10429 & \\
\hline [26] & & $10000 \pm 80$ & $10090 \pm 70$ & & & \\
\hline 27 & & & & & & $14370 \pm 80$ \\
\hline 28 & & & & & & 14300 \\
\hline [29] & $6085 \pm 47 \pm 20$ & $10143 \pm 30 \pm 23$ & $10273 \pm 27 \pm 20$ & $10178 \pm 30 \pm 24$ & $10308 \pm 27 \pm 21$ & $\overline{14366 \pm 9 \pm 2}$ \\
\hline [30] & $6036 \pm 80$ & $10185 \pm 53$ & $10250 \pm 51$ & $10191 \pm 56$ & $10283 \pm 51$ & $14370 \pm 10$ \\
\hline [31] & & $10162 \pm 12$ & & $10184 \pm 12$ & & \\
\hline [32] & 5986,6135 & 10334,10467 & 10397,10606 & 10431,10525 & 10495,10664 & 15023,15175 \\
\hline [33] & & $9960 \pm 90$ & $9970 \pm 90$ & $10300 \pm 200$ & $10400 \pm 200$ & $14300 \pm 200$ \\
\hline [34] & & 10080 & 10100 & & & \\
\hline [35] & & $9940 \pm 910$ & & $10330 \pm 1090$ & & \\
\hline [36] & 6028-6132 & 9998-10137 & 10154-10269 & 10053-10206 & $10228-10355$ & $14444-14688$ \\
\hline [37] & 6096 & 10339,10344 & 10478 & 10468,10473 & 10607 & 15118 \\
\hline [38] & 6096 & 10062 & 10208 & 10101 & 10244 & 14276 \\
\hline [39] & & 10090 & 10180 & 10130 & 10200 & \\
\hline [40] & & 9800 & 9890 & 9840 & 9930 & \\
\hline 411] & & 10185 & 10271 & 10216 & 10289 & \\
\hline [42] & 6079 & 10189 & 10293 & 10218 & 10321 & \\
\hline [45] & & & & & & $14200-15670$ \\
\hline [46] & 6094 & 10314 & 10447 & 10339 & 10467 & \\
\hline [49] & & 10194 & 10267 & & & 14398 \\
\hline [50] & $6044 \pm 18_{-21}^{+20}$ & $10127 \pm 13_{-26}^{+12}$ & $10225 \pm 9_{-13}^{+12}$ & $10151 \pm 14_{-25}^{+16}$ & $10246 \pm 10_{-12}^{+18}$ & \\
\hline [51] & & & & 9858 & 10088 & 14440 \\
\hline$[52]$ & & $10197_{-17}^{+10}$ & $10260_{-34}^{+14}$ & $10236_{-17}^{+9}$ & $10297_{-28}^{+5}$ & \\
\hline [53] & $6069 \pm 34\left(\begin{array}{c}-18 \\
+30\end{array}\right)\left(\begin{array}{l}+35 \\
-0\end{array}\right)$ & $10314 \pm 46_{+11}^{-10}$ & $10365 \pm 40\left(\begin{array}{c}-11 \\
+12\end{array}\right)\left(\begin{array}{l}+16 \\
-0\end{array}\right)$ & $10333 \pm 55_{+6}^{-7}$ & $10383 \pm 39\left(\begin{array}{c}-8 \\
+8\end{array}\right)\left(\begin{array}{l}+12 \\
-0\end{array}\right)$ & \\
\hline [54] & & 10230 & 10320 & 10280 & 10360 & \\
\hline [55] & & 10300 & 10340 & 10340 & 10380 & \\
\hline [56] & & 10160 & 10340 & & & \\
\hline [58] & 6142 & 10440 & 10620 & 10451 & 10628 & 15129 \\
\hline [59] & & 10100 & 10180 & & & \\
\hline$\underline{\underline{60]}}$ & 6090 & & & & & \\
\hline$\underline{\underline{62]}}$ & $6082.8 \pm 5.6$ & & & & & \\
\hline [63] & $6058.9 \pm 8.1$ & & & & & \\
\hline
\end{tabular}




\section{Isospin splitting for the ground $\Xi_{b b}^{*}$ and $\Xi_{b b}$ baryons}

Considering the difference between $u$-quark and $d$-quark (which is the difference between the isospin multiplet), for $\frac{3}{2}^{+}$baryons, Eq. (24) can be expressed as

$$
\begin{gathered}
\left(M_{\Omega_{b b}^{*-}}^{2}-M_{\Xi_{b b}^{* 0}}^{2}\right)+\left(M_{\Xi^{* 0}}^{2}-M_{\Sigma^{*+}}^{2}\right)=\left(M_{\Omega_{b}^{*-}}^{2}-M_{\Sigma_{b}^{*+}}^{2}\right), \\
\left(M_{\Omega_{b b}^{*-}}^{2}-M_{\Xi_{b b}^{*-}}^{2}\right)+\left(M_{\Xi^{*-}}^{2}-M_{\Sigma^{*-}}^{2}\right)=\left(M_{\Omega_{b}^{*-}}^{2}-M_{\Sigma_{b}^{*-}}^{2}\right) .
\end{gathered}
$$

Similarly, considering the isospin breaking effects, for $\frac{1}{2}^{+}$baryons, Eq. (25) can be expressed as

$$
\begin{aligned}
& \left(M_{\Omega_{b b}^{-}}^{2}-M_{\Xi_{b b}^{0}}^{2}\right)+\left(M_{\Xi^{0}}^{2}-M_{\Sigma^{+}}^{2}\right)=\left(M_{\Omega_{b}^{-}}^{2}-M_{\Sigma_{b}^{+}}^{2}\right), \\
& \left(M_{\Omega_{b b}^{-}}^{2}-M_{\Xi_{b b}^{-}}^{2}\right)+\left(M_{\Xi^{-}}^{2}-M_{\Sigma^{-}}^{2}\right)=\left(M_{\Omega_{b}^{-}}^{2}-M_{\Sigma_{b}^{-}}^{2}\right) .
\end{aligned}
$$

From Eq. (28), we can obtain the expression for the isospin splitting $M_{\Xi_{b b}^{*-}}-M_{\Xi_{b b}^{* 0}}$ and get its value to be $1.6 \pm 0.6 \mathrm{MeV}$ (where the uncertainties come from the errors of the input data). From Eq. (29), we can also obtain the expression for the isospin splitting $M_{\Xi_{b b}^{-}}-M_{\Xi_{b b}^{0}}$ and get its value to be $2.3 \pm 0.7 \mathrm{MeV}$.

\section{REGGE PARAMETERS AND MASSES OF THE ORBITALLY EXCITED SINGLY, DOUBLY, AND TRIPLY BOTTOM BARYONS}

In Sec. II, all the masses of unobserved ground state $\frac{1}{2}^{+}$and $\frac{3}{2}^{+}$singly, doubly, and triply bottom baryons were given. In this section, we will calculate the Regge parameters and the masses of the orbitally excited baryons lying on the $\frac{1}{2}^{+}$and $\frac{3}{2}^{+}$bottom baryon trajectories.

\section{A. Regge parameters and Masses of the orbitally excited singly, doubly, and triply} bottom baryons lying on the $\frac{3}{2}^{+}$trajectories

With the help of $\alpha_{n n s}^{\prime}=2 /\left(M_{\Sigma(2030)}^{2}-M_{\Sigma^{*}}^{2}\right)$, from Eq. (17), one can have the expression for $\alpha_{b b s}^{\prime}$ and get its value $\left(\alpha_{b b s}^{\prime}=0.175 \pm 0.014 \mathrm{GeV}^{-2}\right)$. Similarly, using the masses of baryons presented in Eq. (17), with the aid of Eq. (3), one can have the expressions for $\alpha_{b n n}^{\prime}, \alpha_{b s n}^{\prime}, \alpha_{b s s}^{\prime}, \alpha_{b b n}^{\prime}$, and $\alpha_{b b b}^{\prime}$. Their values are given as follows: $\alpha_{b n n}^{\prime}=0.295 \pm 0.022$ $\mathrm{GeV}^{-2}, \alpha_{b s n}^{\prime}=0.294 \pm 0.020 \mathrm{GeV}^{-2}, \alpha_{b s s}^{\prime}=0.292 \pm 0.019 \mathrm{GeV}^{-2}, \alpha_{b b n}^{\prime}=0.176 \pm 0.015$ $\mathrm{GeV}^{-2}, \alpha_{b b b}^{\prime}=0.125 \pm 0.011 \mathrm{GeV}^{-2}$. The results are shown in Table 2. 
Table 2. Regge slopes (in units of $\mathrm{GeV}^{-2}$ ) and Regge intercepts of the ground state $\frac{3}{2}^{+}$ singly, doubly, and triply bottom baryons.

\begin{tabular}{c|cccccc}
\hline \hline & bnn $\left(\Sigma_{b}^{*}\right)$ & bsn $\left(\Xi_{b}^{*}\right)$ & bss $\left(\Omega_{b}^{*}\right)$ & bbn $\left(\Xi_{b b}^{*}\right)$ & bbs $\left(\Omega_{b b}^{*}\right)$ & bbb $\left(\Omega_{b b b}\right)$ \\
\hline$\alpha^{\prime}$ & $0.295 \pm 0.022$ & $0.294 \pm 0.020$ & $0.292 \pm 0.019$ & $0.176 \pm 0.015$ & $0.175 \pm 0.014$ & $0.125 \pm 0.011$ \\
\hline$a(0)$ & $-8.55 \pm 0.74$ & $-8.91 \pm 0.71$ & $-9.26 \pm 0.68$ & $-17.22 \pm 1.44$ & $-17.58 \pm 1.41$ & $-25.89 \pm 2.14$ \\
\hline \hline
\end{tabular}

From Eq. (1), one can have Regge intercepts,

$$
a(0)=J-\alpha^{\prime} M^{2}
$$

For example, with the mass for $\Sigma_{b}^{*}$ and the value of $\alpha_{b n n}^{\prime}$ obtained above, from Eq. (30), $a_{b n n}(0)$ can be given to be $-8.55 \pm 0.74$. Similarly, Regge intercepts $a(0)$ for other $\frac{3}{2}^{+}$ trajectories can be given from Eq. (30). The results are also shown in Table 2. From Eq. (1), one has masses for orbitally excited states,

$$
M_{J}^{2}=[J-a(0)] / \alpha^{\prime}
$$

Then, with the values for $\alpha_{b n n}^{\prime}$ and $a_{b n n}(0)$ obtained above, from Eq. (31), the masses of the orbitally excited baryons ( $L=1,2,3,4$ while $\left.J^{P}=\frac{5}{2}^{-}, \frac{7}{2}^{+}, \frac{9}{2}^{-}, \frac{11}{2}^{+}\right)$lying on the $\Sigma_{b}^{*}$ trajectories can be expressed as functions of masses of the well-established light baryons and singly bottom baryons. The numerical results are shown in Table 3. Similarly, the mass expressions for all the orbitally excited singly and doubly $\frac{3}{2}^{+}$bottom baryons can be extracted. The numerical results are shown in Tables 3 and 4, respectively. (Here and below, masses of orbitally excited baryons are truncated to the $1 \mathrm{MeV}$ digit.) The wave function of a baryon is antisymmetric. For the triply bottom baryon $\Omega_{b b b}$, the flavor wave function is symmetric, the color wave function is antisymmetric. Therefore, its spin-orbital wave function should be symmetric. So, spin-symmetry $\left(S_{3 q}=\frac{3}{2}\right)$ requires orbital-symmetry. Therefore, the oddparity $\Omega_{b b b}$ baryons $(L=1,3)$ can only have the spin-antisymmetry $\left(S_{3 q}=\frac{1}{2}\right)$. This will lead to the real masses lower than our calculated results for such baryons. The above $J^{P}=\frac{5}{2}^{-}, \frac{9}{2}^{-}$ will be changed to $J^{P}=\frac{3}{2}^{-}, \frac{7}{2}^{-}$for $L=1,3 \Omega_{b b b}$ baryons, respectively. The numerical results are shown in Table 5. 
Table 3. The masses of the singly bottom baryons lying on the $\frac{3}{2}^{+}$trajectories (in units of MeV). Our results are labeled with "Pre". The numbers in boldface are the experimental values taken as the input.

\begin{tabular}{|c|c|c|c|c|c|c|c|c|c|c|c|c|c|c|c|}
\hline \multirow[b]{2}{*}{$J^{P}$} & \multicolumn{5}{|c|}{$\Sigma_{b}^{*}$} & \multicolumn{5}{|c|}{$\Xi_{b}^{*}$} & \multicolumn{5}{|c|}{$\Omega_{b}^{*}$} \\
\hline & $\frac{3}{2}^{+}$ & $\frac{5}{2}-$ & $\frac{7}{2}^{+}$ & $\frac{9}{2}^{-}$ & $\frac{11}{2}+$ & $\frac{3}{2}^{+}$ & $\frac{5}{2}-$ & $\frac{7}{2}^{+}$ & $\frac{9}{2}^{-}$ & $\frac{11}{2}+^{+}$ & $\frac{3}{2}^{+}$ & $\frac{5}{2}^{-}$ & $\frac{7}{2}^{+}$ & $\frac{9}{2}^{-}$ & $\frac{11}{2}^{+}$ \\
\hline Pre & $5833.6 \pm 2.4$ & $6117 \pm 20$ & $6388 \pm 38$ & $6648 \pm 55$ & $6898 \pm 71$ & $5952.1 \pm 3.3$ & $6232 \pm 21$ & $6499 \pm 38$ & $6756 \pm 54$ & $7003 \pm 68$ & $6069.4 \pm 6.9$ & $6345 \pm 22$ & $6609 \pm 38$ & $6863 \pm 52$ & $7108 \pm 66$ \\
\hline [1] & $5833.6 \pm 2.4$ & & & & & $5952.1 \pm 3.3$ & & & & & & & & & \\
\hline [8] & 5834 & 6084 & 6260 & 6459 & 6635 & 5963 & 6226 & 6414 & 6610 & 6782 & 6088 & 6334 & 6517 & 6713 & 6884 \\
\hline [16] & $5850 \pm 200$ & & & & & $6020 \pm 170$ & & & & & $6170 \pm 150$ & & & & \\
\hline [17] & 5769 & & & & & 5912 & & & & & 6051 & & & & \\
\hline [20] & 5858 & 6172 & 6333 & & & 5980 & 6201 & 6395 & & & 6102 & 6311 & 6497 & & \\
\hline$[22]$ & 5834 & 6083 & 6262 & & & 5963 & 6218 & 6390 & & & 6088 & 6336 & 6502 & & \\
\hline [24] & $5850 \pm 40$ & & & & & $5980 \pm 40$ & & & & & $6090 \pm 50$ & & & & \\
\hline [46] & 5845 & 6144 & & & & & & & & & 6094 & 6345 & & & \\
\hline [47] & & $5980 \pm 180$ & & & & & $6180 \pm 160$ & & & & & $6430 \pm 130$ & & & \\
\hline [60] & 5844 & & & & & 5967 & & & & & 6090 & & & & \\
\hline [62] & $5832.7 \pm 2$ & & & & & $5959 \pm 4$ & & & & & $6082.8 \pm 5.6$ & & & & \\
\hline [64] & 5805 & 6090 & 6340 & 6540 & & & & & & & & & & & \\
\hline
\end{tabular}

Table 4. The masses of the doubly bottom baryons lying on the $\frac{3}{2}^{+} \Xi_{b b}^{*}$ and $\Omega_{b b}^{*}$ trajectories (in units of $\mathrm{MeV}$ ). Our results are labeled with "Pre".

\begin{tabular}{|c|c|c|c|c|c|c|c|c|c|c|}
\hline \multirow[b]{2}{*}{$J^{P}$} & \multicolumn{5}{|c|}{$\Xi_{b b}^{*}$} & \multicolumn{5}{|c|}{$\Omega_{b b}^{*}$} \\
\hline & $\frac{3}{2}^{+}$ & $\frac{5}{2}$ & $\frac{7}{2}^{+}$ & $\frac{9}{2}$ & $\frac{11}{2}^{+}$ & $\frac{3}{2}^{+}$ & $\frac{5}{2}$ & $\frac{7}{2}^{+}$ & $\frac{9}{2}$ & $\frac{11}{2}^{+}$ \\
\hline Pre & $10316 \pm 38$ & $10588 \pm 59$ & $10853 \pm 80$ & $11112 \pm 99$ & $11365 \pm 118$ & $10431 \pm 40$ & $10700 \pm 60$ & $10964 \pm 80$ & $11221 \pm 99$ & $11472 \pm 117$ \\
\hline [20] & 10367 & 10731 & 10608 & & & 10486 & 10766 & 10732 & & \\
\hline [21] & 10133 & 10580 & 10510 & & & 10257 & 10670 & 10627 & & \\
\hline [22] & 10237 & 10661 & & & & 10389 & 10798 & & & \\
\hline [46] & 10339 & 10759 & & & & 10467 & 10808 & & & \\
\hline [61] & 10352 & 10695 & 11011 & & & & & & & \\
\hline
\end{tabular}

B. Regge parameters and Masses of the orbitally excited singly and doubly baryons lying on the $\frac{1}{2}^{+}$trajectories

For the $\frac{1}{2}^{+}$trajectories, according to the heavy quark symmetry in the heavy quark limit, Regge slopes of $\Sigma_{b}, \Xi_{b}^{\prime}, \Omega_{b}, \Xi_{b b}$, and $\Omega_{b b}$ can be considered to be approximately equal to those of $\Sigma_{b}^{*}, \Xi_{b}^{*}, \Omega_{b}^{*}, \Xi_{b b}^{*}$, and $\Omega_{b b}^{*}$, respectively. According to Refs. [7, 74], $\alpha_{\Lambda_{b}}^{\prime} \simeq \alpha_{\Sigma_{b}}^{\prime}, \alpha_{\Xi_{b}}^{\prime} \simeq \alpha_{\Xi_{b}^{\prime}}^{\prime}$. We take these approximations in this work. Similar to the $\frac{3}{2}^{+}$trajectories, from Eq. (30), one has Regge intercepts $a(0)$ for $\frac{1}{2}^{+}$trajectories. The numerical results are shown in Table 6.

With the values for $a(0)$ and $\alpha^{\prime}$ listed in Table 6, from Eq. (31), the masses of the orbitally excited baryons $\left(L=1,2,3,4\right.$ while $\left.J^{P}=\frac{3}{2}^{-}, \frac{5}{2}^{+}, \frac{7}{2}^{-}, \frac{9}{2}^{+}\right)$lying on the $\frac{1}{2}^{+}$trajectories can be extracted. The numerical results are shown in Tables 7 and 8. 
Table 5. The masses of the triply bottom baryons lying on the $\frac{3}{2}^{+} \Omega_{b b b}$ trajectory (in units of MeV). Our results are labeled with "Pre".

\begin{tabular}{r|lllll}
\hline \hline & \multicolumn{5}{|c}{$\Omega_{b b b}$} \\
$J^{P}$ & $\frac{3}{2}^{+}$ & $\frac{3}{2}^{-}$ & $\frac{7}{2}^{+}$ & $\frac{7}{2}^{-}$ & $\frac{11}{2}^{+}$ \\
\hline Pre & $14788 \pm 80$ & $15055 \pm 101$ & $15318 \pm 123$ & $15577 \pm 143$ & $15831 \pm 163$ \\
\hline$[16]$ & $14830 \pm 100$ & $14950 \pm 110$ & & \\
\hline$[33]$ & $14300 \pm 200$ & $14900 \pm 200$ & & \\
\hline$[20]$ & 14834 & 14976 & 15101 & \\
\hline$[43]$ & 14372 & 14620 & 14800 & \\
\hline$[44]$ & 14372 & 14720 & 14960 & \\
\hline$[48]$ & $14371 \pm 4 \pm 11 \pm 1$ & $14714 \pm 29$ & $14969 \pm 40$ & \\
\hline \hline
\end{tabular}

Table 6. Regge intercepts and Regge slopes (in units of $\mathrm{GeV}^{-2}$ ) of the ground state $\frac{1}{2}^{+}$ singly and doubly bottom baryons.

\begin{tabular}{c|ccccccc}
\hline \hline & bnn $\left(\Lambda_{b}\right)$ & bnn $\left(\Sigma_{b}\right)$ & bsn $\left(\Xi_{b}\right)$ & bsn $\left(\Xi_{b}^{\prime}\right)$ & bss $\left(\Omega_{b}\right)$ & bbn $\left(\Xi_{b b}\right)$ & bbs $\left(\Omega_{b b}\right)$ \\
\hline$\alpha^{\prime}$ & $0.295 \pm 0.022$ & $0.295 \pm 0.022$ & $0.294 \pm 0.020$ & $0.294 \pm 0.020$ & $0.292 \pm 0.019$ & $0.176 \pm 0.015$ & $0.175 \pm 0.014$ \\
\hline$a(0)$ & $-8.82 \pm 0.68$ & $-9.48 \pm 0.73$ & $-9.36 \pm 0.68$ & $-9.85 \pm 0.71$ & $-10.19 \pm 0.69$ & $-17.80 \pm 1.41$ & $-18.17 \pm 1.39$ \\
\hline \hline
\end{tabular}

\section{DISCUSSION AND SUMMARY}

In the present work, we focused on studying masses of the unobserved singly, doubly, and triply bottom baryons. The results for the ground states do not depend on unobservable parameters (such as quark masses and Regge slopes) and distrustful resonances. Regge slopes and Regge intercepts of the singly, doubly, and triply bottom baryon trajectories

Table 7. The masses of the singly bottom baryons lying on the $\frac{1}{2}^{+}$trajectories (in units of MeV). Our results are labeled with "Pre". The numbers in boldface are the experimental values taken as the input.

\begin{tabular}{|c|c|c|c|c|c|c|c|c|c|c|}
\hline \multirow[b]{2}{*}{$J^{P}$} & \multicolumn{5}{|c|}{$\Lambda_{b}$} & \multicolumn{5}{|c|}{$\Xi_{b}$} \\
\hline & $\frac{1}{2}^{+}$ & $\frac{3}{2}^{-}$ & $\frac{5}{2}^{+}$ & $\frac{7}{2}^{-}$ & $\frac{9}{2}^{+}$ & $\frac{1}{2}^{+}$ & $\frac{3}{2}^{-}$ & $\frac{5}{2}^{+}$ & $\frac{7}{2}^{-}$ & $\frac{9}{2}^{+}$ \\
\hline Pre & $5619.51 \pm 0.23$ & $5913 \pm 21$ & $6193 \pm 40$ & $6461 \pm 58$ & $6718 \pm 74$ & $5793.1 \pm 1.8$ & $6080 \pm 19$ & $6354 \pm 37$ & $6616 \pm 53$ & $6869 \pm 68$ \\
\hline [1] & $5619.51 \pm 0.23$ & $5919.73 \pm 0.32$ & & & & $5793.1 \pm 1.8$ & & & & \\
\hline [8] & 5620 & 5942 & 6196 & 6411 & 6599 & 5803 & 6130 & 6373 & 6581 & 6762 \\
\hline [16] & $5650 \pm 200$ & & & & & $5730 \pm 180$ & & & & \\
\hline [20] & 5612 & 5941 & 6183 & & & 5806 & 6093 & 6300 & & \\
\hline [22] & 5622 & 5947 & 6197 & 6405 & & 5812 & 6130 & 6365 & 6558 & \\
\hline [46] & 5618 & 5939 & 6212 & & & & & & & \\
\hline [47] & & $5880 \pm 110$ & & & & & $6070 \pm 130$ & & & \\
\hline [57] & 5619 & 5920 & 6153 & 6351 & 6526 & 5801 & 6106 & 6349 & 6559 & 6747 \\
\hline [60] & 5624 & 5890 & & & & 5825 & 6076 & & & \\
\hline [62] & $5619.7 \pm 1.7$ & $5940 \pm 2$ & & & & $5790-5800$ & $6115 \pm 4$ & & & \\
\hline [64] & 5585 & 5920 & 6165 & 6360 & 6580 & & & & & \\
\hline
\end{tabular}


Table 7. Continued.

\begin{tabular}{|c|c|c|c|c|c|c|c|c|c|c|c|c|c|c|c|}
\hline \multirow[b]{2}{*}{${ }_{J}^{P}$} & \multicolumn{5}{|c|}{$\Sigma_{b}$} & \multicolumn{5}{|c|}{$\Xi_{b}^{\prime}$} & \multicolumn{5}{|c|}{$\Omega_{b}$} \\
\hline & $\frac{1}{2}^{+}$ & $\frac{3}{2}^{-}$ & $\frac{5}{2}^{+}$ & $\frac{7}{2}^{-}$ & $\frac{9}{2}^{+}$ & $\frac{1}{2}^{+}$ & $\frac{3}{2}^{-}$ & $\frac{5}{2}^{+}$ & $\frac{7}{2}^{-}$ & $\frac{9}{2}^{+}$ & $\frac{1}{2}^{+}$ & $\frac{3}{2}^{-}$ & $\frac{5}{2}^{+}$ & $\frac{7}{2}^{-}$ & $\frac{9}{2}^{+}$ \\
\hline Pre & $5813.4 \pm 2.8$ & $6098 \pm 20$ & $6369 \pm 39$ & $6630 \pm 56$ & $6881 \pm 72$ & $5935.0 \pm 0.5$ & $6215 \pm 19$ & $6486 \pm 34$ & $6745 \pm 49$ & $6994 \pm 63$ & $6048.0 \pm 1.9$ & $6325 \pm 18$ & $6590 \pm 34$ & $6844 \pm 48$ & $7090 \pm 62$ \\
\hline [1] & $5813.4 \pm 2.8$ & & & & & $5935.0 \pm 0.5$ & & & & & $6048.0 \pm 1.9$ & & & & \\
\hline [8] & 5808 & 6096 & 6284 & 6500 & 6687 & 5936 & 6234 & 6432 & 6641 & 6821 & 6064 & 6340 & 6529 & 6736 & 6915 \\
\hline [16] & $5800 \pm 190$ & $6000 \pm 180$ & & & & $5960 \pm 170$ & $6140 \pm 140$ & & & & $6110 \pm 160$ & $6260 \pm 150$ & & & \\
\hline [20] & 5833 & 6101 & 6325 & & & 5970 & 6190 & 6393 & & & 6081 & 6304 & 6492 & & \\
\hline [22] & 5805 & 6076 & 6248 & & & 5937 & 6212 & 6377 & & & 6065 & 6330 & 6490 & & \\
\hline [46] & 5823 & 6132 & 6397 & & & & & & & & 6076 & 6336 & 6561 & & \\
\hline [47] & & $5960 \pm 180$ & & & & & $6170 \pm 170$ & & & & & $6430 \pm 130$ & & & \\
\hline [60] & 5789 & 6039 & & & & 5913 & 6157 & & & & 6037 & 6278 & & & \\
\hline [62] & $5611.5 \pm 2$ & $5940 \pm 2$ & & & & $5930 \pm 5$ & & & & & $6052.1 \pm 5.4$ & & & & \\
\hline [64] & 5795 & 6070 & 6325 & 6525 & & & & & & & & & & & \\
\hline
\end{tabular}

Table 8. The masses of the doubly bottom baryons lying on the $\frac{1}{2}^{+} \Xi_{b b}$ and $\Omega_{b b}$ trajectories (in units of MeV). Our results are labelled with "Pre".

\begin{tabular}{|c|c|c|c|c|c|c|c|c|c|c|}
\hline \multirow[b]{2}{*}{$J^{P}$} & \multicolumn{5}{|c|}{$\Xi_{b b}$} & \multicolumn{5}{|c|}{$\Omega_{b b}$} \\
\hline & $\frac{1}{2}^{+}$ & $\frac{3}{2}$ & $\frac{5}{2}^{+}$ & $\frac{7^{2}}{2}$ & $\frac{9}{2}^{+}$ & $\frac{1}{2}^{+}$ & $\frac{3}{2}^{-}$ & $\frac{5}{2}^{+}$ & $\frac{7^{2}}{2}$ & $\frac{9}{2}^{+}$ \\
\hline Pre & $10199 \pm 37$ & $10474 \pm 59$ & $10742 \pm 80$ & $11004 \pm 99$ & $11259 \pm 118$ & $10320 \pm 37$ & $10593 \pm 58$ & $10858 \pm 77$ & $11118 \pm 96$ & $11372 \pm 115$ \\
\hline [16] & $10170 \pm 140$ & $10390 \pm 150$ & & & & $10320 \pm 140$ & $10520 \pm 150$ & & & \\
\hline [20] ] & 10340 & 10495 & 10676 & & & 10454 & 10619 & 10720 & & \\
\hline [21] & 10093 & 10343 & 10497 & & & 10210 & 10462 & & & \\
\hline [22] & 10202 & 10408 & & & & 10359 & 10566 & & & \\
\hline [33] & $9960 \pm 90$ & $10430 \pm 150$ & & & & $9970 \pm 90$ & $10570 \pm 150$ & & & \\
\hline [46] & 10314 & 10476 & 10592 & & & 10447 & 10608 & 10729 & & \\
\hline [61] & 10322 & 10692 & 11002 & & & & & & & \\
\hline
\end{tabular}

were extracted. After that, masses of the orbitally excited $(L=1,2,3,4)$ singly, doubly, and triply bottom baryons were calculated. In this work, all the input masses of baryons used in the calculations were taken from the latest "Review of Particle Physics" [1]. The uncertainties of the results only come from the errors of the input masses. Regge slopes and intercepts used in this work were also calculated from masses of light baryons and singly bottom baryons. No systematic error due to any small deviation from the Regge trajectories has been taken into account in this work.

In Table 1, comparison of the masses of ground state unobserved singly, doubly, and triply bottom baryons extracted in the present work and those given in other references is shown. In Table 2 and Table 6, Regge parameters (slopes and intercepts) were given for the $\frac{3}{2}^{+}$ and $\frac{1}{2}^{+}$trajectories, respectively. In Tables 3, 4, and 5, masses of singly, doubly, and triply bottom baryons lying on the $\frac{3}{2}^{+}$trajectories are shown. In Table 7 and Table 8 , masses of singly and doubly bottom baryons lying on the $\frac{1}{2}^{+}$trajectories are shown, respectively. Our results are neither too big nor too small.

Experimentally, there are two orbitally excited singly bottom baryons: $\Lambda_{b}(5912)$ and $\Lambda_{b}(5920)$ [1], with $J^{P}=\frac{1}{2}^{-}, \frac{3}{2}^{-}$and $M_{\Lambda_{b}(5912)}=5912.11 \pm 0.13 \pm 0.23 \mathrm{MeV}, M_{\Lambda_{b}(5920)}$ 
$=5919.81 \pm 0.23 \mathrm{MeV}$, respectively. $\Lambda_{b}(5912)$ and $\Lambda_{b}(5920)$ were first reported by $\mathrm{LHCb}$ Collaboration [75]. $\Lambda_{b}(5920)$ was confirmed by CDF Collaboration [76]. The mass difference of these two states is small $(<8 \mathrm{MeV})$. This indicates that the spin-orbital couple has small impact on the mass of the singly bottom baryon. In the present work, the mass of the $J^{P}$ $=\frac{3}{2}^{-}(L=1)$ singly bottom baryon is determined to be $5913 \pm 21 \mathrm{MeV}$ (shown in Table 1), which consists with the experimental data.

In Ref. [23], Bjorken pointed out $\frac{M_{\Omega_{b b b}}}{M_{\Upsilon}}=1.56 \pm 0.02$ and gave the mass of $\Omega_{b b b}$ to be $14760 \pm 180 \mathrm{MeV}$, which consists with our result, $M_{\Omega_{b b b}}=14788 \pm 80 \mathrm{MeV}$ (shown in Table 1). In the present work, the central value of mass splittings $\left(M_{\Omega_{b b}^{*}}-M_{\Xi_{b b}^{*}}=10431-10316\right.$ $=115 \mathrm{MeV}, M_{\Omega_{b b}}-M_{\Xi_{b b}}=10320-10199=121 \mathrm{MeV}$ ) are reasonable (close to the usual constituent quark difference $m_{s}-m_{u, d} \approx 120 \mathrm{MeV}$ ). The central values of spin splittings $\left(M_{\Omega_{b b}^{*}}-M_{\Omega_{b b}}=10431-10320=111 \mathrm{MeV}\right.$ and $\left.M_{\Xi_{b b}^{*}}-M_{\Xi_{b b}}=10316-10199=117 \mathrm{MeV}\right)$ are a little big. However, in Refs. [19, 33, 35], the central values of these spin splittings are even bigger than ours (more than $300 \mathrm{MeV}$ ).

The isospin splitting for the $\frac{1}{2}^{+}$doubly bottom baryons in the present work, $M_{\Xi_{b b}^{-}}-M_{\Xi_{b b}^{0}}$ $=2.3 \pm 0.7 \mathrm{MeV}$, is smaller than $5.3 \pm 1.1 \mathrm{MeV}$ in Ref. [77] and $6.3 \pm 1.7 \mathrm{MeV}$ in Ref. [78]. For the $\frac{3}{2}^{+}$doubly bottom baryons, we have also calculated the value of the isospin splitting: $M_{\Xi_{b b}^{*-}}-M_{\Xi_{b b}^{* 0}}=1.6 \pm 0.6 \mathrm{MeV}$. In Ref. [78] strong and electromagnetic sources of isospin breaking are handled separately. Regge theory appears to be a pure QCD emergent phenomenon. In this work, we do not consider the electromagnetic corrections separately because the electromagnetic corrections cancel out in Eqs. (14) and (29). These can be tested by experiments in the future.

In Ref. [8], Ebert et al. gave the values of Regge slopes for singly bottom baryons, which are a little bigger than the corresponding values in this work. For example, $\alpha_{\Xi_{b}}^{\prime}=0.349 \pm$ $0.019 \mathrm{GeV}^{-2}$ in Ref. [8], while $\alpha_{\Xi_{b}}^{\prime}=0.294 \pm 0.020 \mathrm{GeV}^{-2}$ in this work. The mass of the orbitally excited state decreases with the increase of the value of Regge slope. From Tables 3 and 7, one can compare the masses given in Ref. [8] and those given in the present work. The mass differences are small when $L=1,2$.

From Tables 1, 3-5, and 7-8, we can see that the masses of some ground state and orbitally excited singly, doubly, and triply bottom baryons obtained here are in good consistency with the existing experimental data and those given in many other different approaches. However, our predictions for some highly orbitally excited baryons deviate considerably from some predictions in the literature. We expect that our predictions on the masses and Regge parameters in this manuscript can all be tested at $\mathrm{LHCb}$ in the near future. 
In this work, the squared mass relations were used rather than the linear mass relations taken in Refs. [72, 73]. When the mass relations include light baryons, bottom baryons, and doubly bottom baryons (such as Eqs. (14) and (29)), the quadratic mass relations and the linear mass relations lead to significant different values. Besides the addition for baryon spectra, searching for doubly and triply bottom baryons can numerically check the quadratic mass relations and the linear mass relations. The triply bottom baryons $\Omega_{b b b}$ are of considerable theoretical interest [27, 79], since they are free of light quark contamination and may serve as a clean probe to the interplay between perturbative and nonperturbative QCD. Therefore, more efforts should be given to study doubly and triply bottom baryons both theoretically and experimentally.

The mass expressions, Regge parameters, and the masse values in this work would be useful for the discovery of the unobserved singly, doubly, and triply bottom baryon states and the $J^{P}$ assignment of these baryon states when they are observed in the near future.

\section{Acknowledgments}

One of us (Ke-Wei Wei) would like to thank Prof. Bing-Song Zou and Dr. Yao-Feng Zhang for support and discussions. This work was supported in part by National Natural Science Foundation of China (Project No. 11275025, No. U1204115, No. 11305003, No. 11575023, No. 11605009, and No. 11261130311) and Key Project of Scientific and Technological Research of the Education Department of Henan Province (Project No. 12B140001 and No. 13A140014).

[1] K.A. Olive et al. (Particle Data Group), "Review of Particle Physics," Chin. Phys. C, 38, 090001 (2014) and 2015 update.

[2] M. Gell-Mann, "Symmetries of baryons and mesons," Phys. Rev. 125, 1067 (1962); M. GellMann, "A Schematic Model of Baryons and Mesons," Phys. Lett. 8, 214 (1964); S. Okubo, "Note on unitary symmetry in strong interactions," Prog. Theor. Phys. 27, 949 (1962); S. Okubo, "Note on Unitary Symmetry in Strong Interaction. II Excited States of Baryons," Prog. Theor. Phys. 28, 24 (1962).

[3] X.-H. Guo, K.-W. Wei and X.-H. Wu, "Some mass relations for mesons and baryons in Regge phenomenology," Phys. Rev. D 78, 056005 (2008).

[4] K.-W. Wei, B. Chen and X.-H. Guo, "Masses of doubly and triply charmed baryons," Phys. Rev. D 92, no. 7, 076008 (2015) [arXiv:1503.05184 [hep-ph]]. 
[5] A.B. Kaidalov, "Hadronic Mass Relations From Topological Expansion And String Model," Z. Phys. C 12, 63 (1982).

[6] L. Burakovsky, T. Goldman, and L.P. Horwitz, "New mass relations for heavy quarkonia," Phys. Rev. D 56, 7119 (1997); L. Burakovsky, T. Goldman, and L.P. Horwitz, "New mass relation for meson 25-plet," J. Phys. G 24, 771 (1998).

[7] L. Burakovsky, T. Goldman, and L.P. Horwitz, "New quadratic baryon mass relations," Phys. Rev. D 56, 7124 (1997).

[8] D. Ebert, R.N. Faustov and V.O. Galkin, "Spectroscopy and Regge trajectories of heavy baryons in the relativistic quark-diquark picture," Phys. Rev. D 84, 014025 (2011).

[9] D. Ebert, R. N. Faustov and V.O. Galkin, "Mass spectra and Regge trajectories of light mesons in the relativistic quark model," Phys. Rev. D 79, 114029 (2009); D. Ebert, R.N. Faustov and V.O. Galkin, "Heavy-light meson spectroscopy and Regge trajectories in the relativistic quark model," Eur. Phys. J. C 66, 197 (2010).

[10] K.-W. Wei and X.-H. Guo, "Mass spectra of doubly heavy mesons in Regge phenomenology," Phys. Rev. D 81, 076005 (2010); K.-W. Wei, X.-P. Dong and G. Lu, "Masses of $s \bar{s}$ states and nonet mixing angles," Int. J. Mod. Phys. A 26, 2065 (2011).

[11] D.-M. Li, B. Ma, Y.-X. Li, Q.-K. Yao, and H. Yu, "Meson spectrum in Regge phenomenology," Eur. Phys. J. C 37, 323 (2004).

[12] A.V. Anisovich, V.V. Anisovich, and A.V. Sarantsev, "Systematics of $q \bar{q}$ states in the $\left(n, M^{2}\right)$ and (J, $\left.M^{2}\right)$ planes," Phys. Rev. D 62, 051502(R) (2000); V.V. Anisovich, "Systematics of quark antiquark states and scalar exotic mesons," Phys. Usp. 47, 45 (2004) [Usp. Fiz. Nauk 174, 49 (2004)].

[13] A. Zhang, "Charmonium spectrum and new observed states," Phys. Lett. B 647, 140 (2007).

[14] M.M. Brisudova, L. Burakovsky, and T. Goldman, "Effective functional form of Regge trajectories," Phys. Rev. D 61, 054013 (2000).

[15] P. Masjuan, E.R. Arriola and W. Broniowski, "Systematics of radial and angular-momentum Regge trajectories of light non-strange $q \bar{q}$-states," Phys. Rev. D 85, 094006 (2012).

[16] Z.G. Wang, "Analysis of the $\frac{3}{2}^{+}$heavy and doubly heavy baryon states with QCD sum rules," Eur. Phys. J. C 68, 459 (2010); Z.G. Wang, "Analysis of the $\frac{1}{2}^{+}$doubly heavy baryon states with QCD sum rules," Eur. Phys. J. A 45, 267 (2010); Z.G. Wang, "Analysis of the Triply Heavy Baryon States with QCD Sum Rules," Commun. Theor. Phys. 58, 723 (2012); Z.G. Wang, "Analysis of the $\frac{1}{2}^{-}$and $\frac{3}{2}^{-}$heavy and doubly heavy baryon states with QCD sum rules," Eur. Phys. J. A 47, 81 (2011). Z.G. Wang, "Analysis of the $\frac{1}{2}^{ \pm}$antitriplet heavy baryon states with QCD sum rules," Eur. Phys. J. C 68, 479 (2010); Z.G. Wang, "Reanalysis of the heavy baryon states $\Omega_{b}, \Omega_{c}, \Xi_{b}^{\prime}, \Xi_{c}^{\prime}, \Sigma_{b}$ and $\Sigma_{c}$ with QCD sum rules," Phys. Lett. B 685, 59 (2010)

[17] W.A. Ponce, "Heavy Quarks In A Spherical Bag," Phys. Rev. D 19, 2197 (1979).

[18] A.P. Martynenko, "Ground-state triply and doubly heavy baryons in a relativistic three-quark model," Phys. Lett. B 663, 317 (2008).

[19] J.R. Zhang and M.Q. Huang, "Heavy baryon spectroscopy in QCD," Phys. Rev. D 78, 094015 (2008); J.R. Zhang and M.Q. Huang, "Doubly heavy baryons in QCD sum rules," Phys. Rev. D 78, 094007 (2008); J.R. Zhang and M.Q. Huang, "Deciphering triply heavy baryons in 
terms of QCD sum rules," Phys. Lett. B 674, 28 (2009).

[20] W. Roberts and M. Pervin, "Heavy Baryons in a Quark Model," Int. J. Mod. Phys. A 23, 2817 (2008) [arXiv:0711.2492 [nucl-th]].

[21] S.S. Gershtein, V.V. Kiselev, A.K. Likhoded, and A.I. Onishchenko, "Spectroscopy of doubly heavy baryons," Phys. Rev. D 62, 054021 (2000); V.V. Kiselev, A.K. Likhoded, O.N. Pakhomova, and V.A. Saleev, "Mass spectra of doubly heavy $\Omega_{Q Q^{\prime}}$ baryons," Phys. Rev. D 66, 034030 (2002).

[22] D. Ebert, R.N. Faustov and V.O. Galkin, "Masses of heavy baryons in the relativistic quark model," Phys. Rev. D 72, 034026 (2005); D. Ebert, R.N. Faustov, V.O. Galkin, and A.P. Martynenko, "Mass spectra of doubly heavy baryons in the relativistic quark model," Phys. Rev. D 66, 014008 (2002); D. Ebert, R.N. Faustov and V.O. Galkin, "Masses of excited heavy baryons in the relativistic quark model," Phys. Lett. B 659, 612 (2008).

[23] J.D. Bjorken, "Is the CCC a New Deal for Baryon Spectroscopy?" AIP Conf. Proc. 132, 390 (1985) [FERMILAB-CONF-85/069].

[24] R. Roncaglia, D.B. Lichtenberg, and E. Predazzi, "Predicting The Masses Of Baryons Containing One Or Two Heavy Quarks," Phys. Rev. D 52, 1722 (1995).

[25] D.-H. He, K. Qian, Y.-B. Ding, X.-Q. Li, and P.-N. Shen, "Evaluation of spectra of baryons containing two heavy quarks in bag model," Phys. Rev. D 70, 094004 (2004).

[26] V.V. Kiselev and A.E. Kovalsky, "Doubly heavy baryons $\Omega_{Q Q^{\prime}}$ versus $\Xi_{Q Q^{\prime}}$ in sum rules of NRQCD," Phys. Rev. D 64, 014002 (2001).

[27] Y. Jia, "Variational study of weakly coupled triply heavy baryons," JHEP 0610, 073 (2006).

[28] P. Hasenfratz, R.R. Horgan, J. Kuti and J.M. Richard, "Heavy Baryon Spectroscopy In The QCD Bag Model," Phys. Lett. B 94, 401 (1980).

[29] Z.S. Brown, W. Detmold, S. Meinel and K. Orginos, "Charmed bottom baryon spectroscopy from lattice QCD," Phys. Rev. D 90, no. 9, 094507 (2014).

[30] Z.S. Brown, W. Detmold, S. Meinel and K. Orginos, "Charmed Bottom Baryon Spectroscopy," PoS LATTICE 2013, 248 (2014).

[31] M. Karliner and J.L. Rosner, "Baryons with two heavy quarks: Masses, production, decays, and detection," Phys. Rev. D 90, 094007 (2014).

[32] Z. Ghalenovi, A.A. Rajabi, S.x. Qin and D.H. Rischke, "Ground-State Masses and Magnetic Moments of Heavy Baryons," Mod. Phys. Lett. A 29, 1450106 (2014).

[33] T.M. Aliev, K. Azizi and M. Savci, "The masses and residues of doubly heavy spin-3/2 baryons," J. Phys. G 40, 065003 (2013); T.M. Aliev, K. Azizi and M. Savci, "Doubly Heavy Spin-1/2 Baryon Spectrum in QCD," Nucl. Phys. A 895, 59 (2012); T.M. Aliev, K. Azizi and M. Savci, "Properties of triply heavy spin-3/2 baryons," J. Phys. G 41, 065003 (2014).

[34] M.-H. Weng, X.-H. Guo and A.W. Thomas, "Bethe-Salpeter equation for doubly heavy baryons in the covariant instantaneous approximation," Phys. Rev. D 83, 056006 (2011).

[35] E. Bagan, M. Chabab and S. Narison, "Baryons with two heavy quarks from QCD spectral sum rules," Phys. Lett. B 306, 350 (1993).

[36] B. Patel, A. Majethiya and P.C. Vinodkumar, "Masses and Magnetic moments of Triply Heavy Flavour Baryons in Hypercentral Model," Pramana 72, 679 (2009); B. Patel, A.K. Rai and P.C. Vinodkumar, "Heavy Flavour Baryons in Hyper Central Model," Pramana 70, 797 
(2008).

[37] Z. Ghalenovi, A.A. Rajabi and M. Hamzavi, "The heavy baryon masses in variational approach and spin-isospin dependence," Acta Phys. Polon. B 42, 1849 (2011).

[38] A. Bernotas and V. Simonis, "Heavy hadron spectroscopy and the bag model," Lith. J. Phys. 49, 19 (2009).

[39] V.V. Kiselev and A.K. Likhoded, "Baryons with two heavy quarks," Phys. Usp. 45, 455 (2002) [Usp. Fiz. Nauk 172, 497 (2002)].

[40] L. Tang, X.H. Yuan, C.F. Qiao and X.Q. Li, "Study of Doubly Heavy Baryon Spectrum via QCD Sum Rules," Commun. Theor. Phys. 57, 435 (2012).

[41] F. Giannuzzi, "Doubly heavy baryons in a Salpeter model with AdS/QCD inspired potential," Phys. Rev. D 79, 094002 (2009).

[42] A. Valcarce, H. Garcilazo and J. Vijande, "Towards an understanding of heavy baryon spectroscopy," Eur. Phys. J. A 37, 217 (2008).

[43] J. Vijande, A. Valcarce and H. Garcilazo, "Heavy-baryon quark model picture from lattice QCD," Phys. Rev. D 90, no. 9, 094004 (2014).

[44] J. Vijande, A. Valcarce and H. Garcilazo, "Constituent-quark model description of triply heavy baryon nonperturbative lattice QCD data," Phys. Rev. D 91, no. 5, 054011 (2015).

[45] F.J. Llanes-Estrada, O.I. Pavlova and R. Williams, "A First Estimate of Triply Heavy Baryon Masses from the pNRQCD Perturbative Static Potential," Eur. Phys. J. C 72, 2019 (2012).

[46] T. Yoshida, E. Hiyama, A. Hosaka, M. Oka and K. Sadato, "Spectrum of heavy baryons in the quark model," Phys. Rev. D 92, no. 11, 114029 (2015)

[47] Q. Mao, H.-X. Chen, W. Chen, A. Hosaka, X. Liu and S.-L. Zhu, "QCD sum rule calculation for P-wave bottom baryons," Phys. Rev. D 92, no. 11, 114007 (2015)

[48] S. Meinel, "Prediction of the $\Omega_{b b b}$ mass from lattice QCD," Phys. Rev. D 82, 114514 (2010); S. Meinel, "Excited-state spectroscopy of triply-bottom baryons from lattice QCD," Phys. Rev. D 85, 114510 (2012).

[49] B. Silvestre-Brac, "Spectrum and static properties of heavy baryons," Few Body Syst. 20, 1 (1996).

[50] R. Lewis and R.M. Woloshyn, "Bottom baryons from a dynamical lattice QCD simulation," Phys. Rev. D 79, 014502 (2009)

[51] W. Zheng and H.R. Pang, "Momentum-space Faddeev calculations for ground-state triply and doubly heavy baryons in the constituent quark model," Mod. Phys. Lett. A 25, 2077 (2010).

[52] C. Albertus, E. Hernandez, J. Nieves and J.M. Verde-Velasco, "Static properties and semileptonic decays of doubly heavy baryons in a nonrelativistic quark model," Eur. Phys. J. A 32, 183 (2007).

[53] A. Ali Khan, T. Bhattacharya, S. Collins, C.T.H. Davies, R. Gupta, C. Morningstar, J. Shigemitsu and J.H. Sloan, "Heavy-light mesons and baryons with $b$ quarks," Phys. Rev. D 62, 054505 (2000)

[54] D. Ebert, R.N. Faustov, V.O. Galkin, A.P. Martynenko and V.A. Saleev, "Heavy baryons in the relativistic quark model," Z. Phys. C 76, 111 (1997).

[55] S.P. Tong, Y.B. Ding, X.H. Guo, H.Y. Jin, X.Q. Li, P.N. Shen and R. Zhang, "Spectra of baryons containing two heavy quarks in potential model," Phys. Rev. D 62, 054024 (2000). 
[56] I.M. Narodetskii and M.A. Trusov, "The Heavy baryons in the nonperturbative string approach," Phys. Atom. Nucl. 65, 917 (2002) [Yad. Fiz. 65, 949 (2002)].

[57] B. Chen, K.-W. Wei and A. Zhang, "Assignments of $\Lambda_{Q}$ and $\Xi_{Q}$ baryons in the heavy quarklight diquark picture," Eur. Phys. J. A 51, 82 (2015).

[58] R. Dhir, C.S. Kim and R.C. Verma, "Magnetic Moments of Bottom Baryons: Effective mass and Screened Charge," Phys. Rev. D 88, 094002 (2013).

[59] A. Faessler, T. Gutsche, M.A. Ivanov, J.G. Korner, V.E. Lyubovitskij, D. Nicmorus and K. Pumsa-ard, "Magnetic moments of heavy baryons in the relativistic three-quark model," Phys. Rev. D 73, 094013 (2006).

[60] H. Garcilazo, J. Vijande and A. Valcarce, "Faddeev study of heavy baryon spectroscopy," J. Phys. G 34, 961 (2007).

[61] B. Eakins and W. Roberts, "Symmetries and Systematics of Doubly Heavy Hadrons," Int. J. Mod. Phys. A 27, 1250039 (2012).

[62] M. Karliner, B. Keren-Zur, H.J. Lipkin and J.L. Rosner, "The Quark Model and $b$ Baryons," Annals Phys. 324, 2 (2009).

[63] E.E. Jenkins, "Model-Independent Bottom Baryon Mass Predictions in the 1/N(c) Expansion," Phys. Rev. D 77, 034012 (2008).

[64] S. Capstick and N. Isgur, "Baryons In A Relativized Quark Model With Chromodynamics," Phys. Rev. D 34, 2809 (1986).

[65] L. Burakovsky and T. Goldman, "On the Regge slopes intramultiplet relation," Phys. Lett. B 434, 251 (1998).

[66] A. Capella and J. Tran Thanh van, "Hadron-Nucleus Interactions And The Leading Particle Effect In A Dual-Parton Model," Z. Phys. C 10, 249 (1981); A.B. Kaidalov and K.A. TerMartirosian, "Multiple Production Of Hadrons At High-Energies In The Model Of QuarkGluon Strings," Sov. J. Nucl. Phys. 39, 979 (1984) [Yad. Fiz. 39, 1545 (1984)]; G.I. Lykasov and M.N. Sergeenko, "Inclusion of transverse quark momenta in the model of quark - gluon strings," Z. Phys. C 52, 635 (1991).

[67] K.P. Das and R.C. Hwa, "Quark-antiquark recombination in the Fragmentation Region," Phys. Lett. B 68, 459 (1977); A.V. Batunin, A.K. Likhoded, and V.V. Kiselev, "Are the Gluon Distributions Different in $\pi$ and $K$ Mesons?," Sov. J. Nucl. Phys. 49, 346 (1989) [Yad. Fiz. 49, 554 (1989)]; T. Tashiro et al., "Single Particle Spectra in Soft Hadron Hadron Interactions and Two Component Cascade Model," Z. Phys. C 35, 21 (1987).

[68] J.L. Basdevant and S. Boukraa, "Successes and Difficulties of Unified Quark-Antiquark Potential Models," Z. Phys. C 28, 413 (1985); J.L. Basdevant and S. Boukraa, "Baryon Masses In Relativistic Potential Models," Z. Phys. C 30, 103 (1986).

[69] P.D. Collins, "An introduction to Regge theory and high energy physics," Cambridge University Press, 1977.

[70] T. Regge, "Introduction to complex orbital momenta," Nuovo Cim. 14, 951 (1959); T. Regge, "Bound states, shadow states and Mandelstam representation," Nuovo Cim. 18, 947 (1960).

[71] G.F. Chew and S.C. Frautschi, "Principle of Equivalence for All Strongly Interacting Particles Within the S Matrix Framework," Phys. Rev. Lett. 7, 394 (1961); G.F. Chew and S.C. Frautschi, "Regge Trajectories and the Principle of Maximum Strength for Strong Interac- 
tions," Phys. Rev. Lett. 8, 41 (1962).

[72] S. Iwao, "Mass formula for hadrons with zero Pontryagin index," Prog. Theor. Phys. 87, 1443 (1992).

[73] A.W. Hendry and D.B. Lichtenberg, "Hadron Mass Relations in a Charmed Quark Model with Broken SU(8) Symmetry," Phys. Rev. D 12, 2756 (1975).

[74] N.A. Kobylinsky, A.I. Kosenko and R.S. Tutik, "Interrelations Between Baryon Trajectories And New Mass Formulas For Baryon Octet," Kiev Institute of Theoretical Physics Report No. ITF-79-36E, 1979.

[75] R. Aaij et al. [LHCb Collaboration], "Observation of excited $\Lambda_{b}^{0}$ baryons," Phys. Rev. Lett. 109, 172003 (2012).

[76] T.A. Aaltonen et al. [CDF Collaboration], "Evidence for a bottom baryon resonance $\Lambda_{b}^{* 0}$ in CDF data," Phys. Rev. D 88, no. 7, 071101 (2013).

[77] C.-W. Hwang and C.-H. Chung, "Isospin mass splittings of heavy baryons in HQS," Phys. Rev. D 78, 073013 (2008).

[78] S.J. Brodsky, F.-K. Guo, C. Hanhart and U.G. Meissner, "Isospin splittings of doubly heavy baryons," Phys. Lett. B 698, 251 (2011).

[79] N. Brambilla, F. Karbstein and A. Vairo, "Symmetries of the three-heavy-quark system and the color-singlet static energy at next-to-next-to-leading logarithmic order," Phys. Rev. D 87, no. 7, 074014 (2013). 